\section{RSP}

http://www.rsp.fsp.usp.br/
Revista de Saúde Pública

\title{
Acesso à Atenção Primária à Saúde por idosos residentes em zona rural no Sul do Brasil
}

\author{
Luiza Santos Ferreira' iD, Laísa Rodrigues Moreira" (iD, Simone dos Santos Paludo"I iD, Rodrigo \\ Dalke Meuccilv iD \\ ' Universidade Federal do Rio Grande. Faculdade de Medicina. Programa de Pós-Graduação em Saúde Pública. \\ Rio Grande, RS, Brasil \\ " Universidade Federal de Pelotas. Faculdade de Medicina. Programa de Pós-Graduação em Epidemiologia. \\ Pelotas, RS, Brasil \\ II' Universidade Federal do Rio Grande. Instituto de Ciências Humanas e da Informação. Rio Grande, RS, Brasil \\ iv Universidade Federal do Rio Grande. Faculdade de Medicina. Rio Grande, RS, Brasil
}

\footnotetext{
Correspondência:

Luiza Santos Ferreira

R. General Osório, s/n

96203-900 Rio Grande, RS, Brasil

E-mail: luferreira.psi@gmail.com

Recebido: 8 jan 2020

Aprovado: 19 mai 2020

Como citar: Ferreira LS, Moreira LR, Paludo SS, Meucci RD. Acesso à Atenção Primária à Saúde por idosos residentes em zona rural no Sul do Brasil. Rev Saude Publica. 2020;54:149.

Copyright: Este é um artigo de acesso aberto distribuído sob os termos da Licença de Atribuição Creative Commons, que permite uso irrestrito, distribuição e reprodução em qualquer meio, desde que o autor e a fonte originais sejam creditados.
}

\section{RESUMO}

OBJETIVO: Caracterizar o acesso e utilização de serviços de saúde considerados como referência pela população rural idosa de um município do sul do Brasil, com cobertura de $100 \%$ da área rural pela Estratégia Saúde da Família (ESF), investigando os fatores associados à escolha da Unidade Básica de Saúde da Família (UBSF) como serviço de referência.

MÉTODOS: Estudo transversal com amostragem sistemática dos domicílios da área rural do município de Rio Grande (RS), realizado em 2017, através de questionário padronizado, aplicado em domicílio. Foram realizadas análises descritivas referentes a perfil sociodemográfico; tipo de serviço de referência escolhido; e motivos de escolha/utilização do serviço de primeira opção e da UBSF mais próxima. Realizou-se regressão de Poisson para investigar fatores associados ao tipo de serviço de referência escolhido.

RESULTADOS: Participaram do estudo 1.030 idosos, dos quais 61,4\% indicaram a unidade básica como serviço de primeira opção/referência. Enquanto aqueles que escolheram ser atendidos na UBSF o faziam principalmente em razão da proximidade (82,6\%), os demais procuraram outros locais pela percepção de maior facilidade $(34,6 \%$ ) e resolutividade $(52,6 \%)$. Quase $2 / 3$ dos entrevistados buscaram atendimento na UBSF no último ano, e os motivos foram diferentes entre aqueles que consideravam a unidade como referência (doença crônica) e aqueles que procuravam outro local (procedimentos). Além disso, quanto menor a idade, renda, escolaridade e distância residência-unidade, maior a probabilidade do idoso considerar a UBSF mais próxima como serviço de referência.

CONCLUSÃO: A ESF tem conseguido atingir a parcela rural idosa em situação de vulnerabilidade, indo ao encontro de um sistema de saúde público equitativo. Todavia, novas avaliações para averiguar adequação e qualidade do atendimento se fazem necessárias, visto que estrutura social, fatores capacitantes (como condição econômica) e possíveis crenças sobre saúde ainda definem o padrão de escolha do serviço.

DESCRITORES: Idoso. Acesso aos Serviços de Saúde. Atenção Primária à Saúde. Estratégia Saúde da Família. Saúde da População Rural. 


\section{INTRODUÇÃO}

A atenção em saúde vem se reconstruindo ao longo do tempo, através de conceitos e práticas mais abrangentes. Com a Declaração de Alma-Ata ${ }^{1}$, mais foco e importância foram dados internacionalmente à atenção primária em saúde (APS), que no Brasil está sendo desenvolvida através da Estratégia Saúde da Família (ESF), trabalho multiprofissional, porta de entrada ao sistema de saúde, dirigido a populações de territórios definidos, pelas quais uma equipe assume responsabilidade sanitária ${ }^{2-5}$. Atualmente, $64,34 \%$ da população brasileira é atendida pela ESF${ }^{6}$. Mesmo com dificuldades no processo de mudança das unidades básicas tradicionais para a ESF, o número de pessoas que acessa a atenção básica aumentou cerca de $450 \%$ entre 1981 e $2008^{7}$.

Na constante ampliação e reestruturação dos sistemas de saúde, a avaliação é essencial, e a forma como se dá o acesso dos usuários aos serviços é um dos aspectos fundamentais desta análise ${ }^{5,8-10}$. O conceito de "acesso" é complexo, e não há unanimidade entre os estudiosos da área. A definição do termo varia conforme tempo e contexto, prevalecendo, porém, um significado associado ao desempenho dos sistemas de saúde quanto a sua capacidade de oferta ${ }^{11}$.

A avaliação do acesso é ainda mais importante quando se trata de populações que tradicionalmente enfrentam dificuldades para obter a devida atenção em saúde, como é o caso da população idosa residente em área rural ${ }^{12-14}$. Por apresentar maiores necessidades, esse grupo tende a usar mais os serviços de saúde ${ }^{8,15}$, e dificuldades no acesso ao atendimento adequado podem trazer agravos. Estudos que comparam a utilização dos serviços de atenção primária quanto à localização mostram menos uso e/ou pior qualidade na área rural em comparação à urbana ${ }^{4,8,12-16}$. A baixa variedade ou qualidade dos serviços disponíveis localmente, por sua vez, pode levar à demora na busca por tratamento ou à busca por serviços mais distantes, causando maior desgaste físico, emocional e financeiro ${ }^{17}$.

Apesar da vulnerabilidade da população idosa em área rural e do complexo processo de definição de um plano de cuidado, poucos estudos têm direcionado esforços a essa parcela da população $0^{4,18}$. No entanto, uma vez que esse grupo apresenta necessidades e limitações específicas, é pertinente investigar como se dá sua busca por serviços de saúde e possíveis dificuldades encontradas. Assim, o presente estudo objetiva caracterizar o acesso e utilização de serviços de saúde considerados de referência pela população rural idosa de um município do sul do Brasil com cobertura de $100 \%$ da área rural pela ESF, investigando fatores de escolha da Unidade Básica de Saúde da Família (UBSF) como serviço de referência.

\section{MÉTODOS}

Esta pesquisa conta com dados referentes ao perfil de acesso e utilização de serviços de saúde por idosos (indivíduos com 60 anos ou mais) residentes na área rural de Rio Grande (RS). Os dados representam um recorte do estudo transversal "Saúde da População Rural Rio-Grandina”, realizado pelo Programa de Pós-Graduação em Saúde Pública da Universidade Federal do Rio Grande (FURG), que investigou indicadores básicos de saúde e o padrão de morbidade, acesso e utilização de serviços de saúde em crianças com até 5 anos de idade incompletos, mulheres em idade fértil e idosos moradores da área rural de Rio Grande.

O município de Rio Grande fica no estado do Rio Grande do Sul, a $350 \mathrm{~km}$ da capital Porto Alegre, e conta com aproximadamente 200 mil habitantes. Destes, 5,5\% (cerca de 8.225 indivíduos) residiam em área rural no momento do estudo transversal, e dentre os moradores rurais $13,1 \%$ eram idosos ${ }^{19}$. Quanto à rede pública de saúde, o município contava com a cobertura de 53\% de seu território total pela ESF, chegando a 100\% na área rural. Encontravam-se disponíveis à população rural: 10 equipes de saúde da família, instaladas em oito UBSF (uma delas aberta 24 horas, na forma de pronto atendimento) apoiadas por dois Núcleos Ampliados de Saúde da Família (NASF). 
Realizou-se cálculo de tamanho de amostra mínimo para estimar a prevalência da variável dependente (considerando nível de confiança de $95 \%$, frequência de $70 \%$ para a estimativa do desfecho, margem de erro de 3 pontos percentuais e efeito de delineamento de 1,5) e fatores associados a ela (considerando poder de $80 \%$, acréscimo de $10 \%$ para perdas e recusas, e $15 \%$ para controle de fatores de confusão), chegando ao número de 857 indivíduos.

Os domicílios dos 23 setores censitários rurais do município foram abordados entre abril e novembro de 2017, com um intervalo sistemático de um domicílio a cada quatro amostrados, totalizando a amostragem de aproximadamente $80 \%$ das populações de interesse. Domicílios sem indivíduos nas faixas etárias de interesse não foram considerados elegíveis. Por outro lado, quando os residentes dos domicílios amostrados se enquadravam nas faixas etárias de interesse, todos os indivíduos elegíveis eram entrevistados. Foram excluídos da pesquisa apenas idosos que se encontravam institucionalizados no período de realização das entrevistas. Nos casos de idosos com incapacidade física ou mental para responder o questionário, o cuidador foi entrevistado.

O instrumento utilizado para coleta de dados foi um questionário padronizado. Investigou-se o perfil de utilização de acordo com o serviço considerado como de referência pelo entrevistado, através da pergunta: "Quando o(a) senhor(a) precisa de um atendimento de saúde, qual é o primeiro serviço a que o(a) senhor(a) vai?”. As respostas foram posteriormente categorizadas em "UBSF de referência" (sendo esta a unidade mais próxima da residência do participante) ou "outro serviço", incluindo hospitais, consultórios médicos particulares e conveniados a plano de saúde privados e serviços localizados em outros municípios.

As variáveis independentes consideradas foram: sexo (masculino; feminino), idade (60 a 69 anos; 70 a 79 anos; 80 anos ou mais), cor da pele (branca ou preta/parda/amarela/indígena), escolaridade ( 0 a 4 anos; 5 a 8 anos; 9 anos ou mais), renda familiar (em tercis), morar sozinho (não ou sim), possuir plano de saúde (não ou sim) e distância da UBSF mais próxima (< $1 \mathrm{~km}$; 1 a 2,9 km; 3 a 5,9 km; 6 a 9,9 km; $10 \mathrm{~km}$ ou mais). Além disso, as seguintes variáveis relacionadas à situação de saúde também foram investigadas: possuir doença crônica (não ou sim) e estado de saúde autorreferido (bom/muito bom; regular; ruim/muito ruim).

Através do programa estatístico Stata 14.1, foram realizadas análises estatísticas descritivas do perfil sociodemográfico da amostra, do tipo de serviço de referência escolhido e dos motivos de escolha e de sua utilização. Além disso, foi realizada regressão de Poisson, com ajuste robusto para variância, para investigar fatores associados ao tipo de serviço de referência escolhido.

Para a análise ajustada, foi construído modelo conceitual hierarquizado por níveis ${ }^{20}$, considerando as três categorias de fatores identificadas por Aday e Andersen ${ }^{21}$ como influenciadoras do acesso aos serviços de saúde ${ }^{9}$ fatores predisponentes (características individuais, prévias ao problema de saúde, que influenciam na propensão do sujeito a utilizar os serviços de saúde), fatores capacitantes (meios que possibilitam a obtenção do atendimento) e necessidades em saúde (relacionadas ao nível de doença). Desse modo, o modelo utilizado nas análises foi: 1) nível distal: fatores predisponentes (sexo, idade e cor da pele); 2) nível intermediário: fatores capacitantes (escolaridade, renda familiar, morar sozinho, possuir plano de saúde e distância da UBSF mais próxima); 3) nível proximal: necessidades em saúde (doença crônica e estado de saúde autorreferido). Foi realizado ajuste para possíveis fatores de confusão do mesmo nível ou superior, considerando o valor $\mathrm{p}<0,20$ como limite para manter as variáveis no modelo final, a fim de controlar para confusões positivas.

Quanto aos aspectos éticos, o projeto de pesquisa foi aprovado pelo Comitê de Ética em Pesquisa da Universidade Federal do Rio Grande sob o Parecer no 51/2017, Processo 23116.009484/2016-26. Todos os idosos entrevistados, ou cuidadores (em caso de limitação do participante), assinaram o termo de consentimento livre e esclarecido. 


\section{RESULTADOS}

Dos 1.351 idosos moradores da área rural de Rio Grande, 83,7\% (n = 1.131) foram amostrados para o estudo, chegando a um número final de 1.030 idosos entrevistados $(8,9 \%$ de perdas e recusas). A amostra foi formada majoritariamente por homens, entre 60 e 69 anos de idade, cor da pele branca, 0 a 4 anos de escolaridade, e que moravam pelo menos com mais uma pessoa na residência. Somente $1 / 3$ dos participantes tinha plano de saúde privado, e apenas 14,9\% dos idosos moravam a menos de um quilômetro de distância da UBSF mais próxima (Tabela 1). A renda domiciliar mensal mediana foi de $\mathrm{R} \$ 1.874,00(\mathrm{IQQ}=\mathrm{R} \$ 1.000,00-\mathrm{R} \$ 2.000,00)$.

Tabela 1. Descrição da amostra de idosos moradores da área rural do município de Rio Grande, Rio Grande do Sul, Brasil, $2017(n=1.030)$.

\begin{tabular}{|c|c|c|}
\hline Variável & $\mathbf{n}$ & $\%$ \\
\hline \multicolumn{3}{|l|}{$\operatorname{Sexo}(n=1.030)$} \\
\hline Masculino & 568 & 55,15 \\
\hline Feminino & 462 & 44,85 \\
\hline \multicolumn{3}{|l|}{ Idade $(\mathrm{n}=1.029)$} \\
\hline 60 a 69 anos & 529 & 51,41 \\
\hline 70 a 79 anos & 327 & 31,78 \\
\hline 80 anos ou mais & 173 & 16,81 \\
\hline \multicolumn{3}{|l|}{ Cor da pele $(n=1.028)$} \\
\hline Branca & 942 & 91,63 \\
\hline Preta/Parda/Amarela/Indígena & 86 & 8,37 \\
\hline \multicolumn{3}{|l|}{ Escolaridade $(\mathrm{n}=1.017)$} \\
\hline 0 a 4 anos & 703 & 69,13 \\
\hline 5 a 8 anos & 233 & 22,91 \\
\hline 9 anos ou mais & 81 & 7,96 \\
\hline \multicolumn{3}{|l|}{ Mora sozinho $(\mathrm{n}=1.030)$} \\
\hline Não & 797 & 77,38 \\
\hline Sim & 233 & 22,62 \\
\hline \multicolumn{3}{|l|}{ Distância domicílio-UBSF ( $\mathrm{n}=842)$} \\
\hline$<1 \mathrm{~km}$ & 125 & 14,85 \\
\hline $1 \mathrm{~km}-2,9 \mathrm{~km}$ & 175 & 20,78 \\
\hline $3 \mathrm{~km}-4,9 \mathrm{~km}$ & 101 & 12,00 \\
\hline 5 km-9,9 km & 259 & 30,75 \\
\hline $10 \mathrm{~km}$ ou mais & 182 & 21,62 \\
\hline \multicolumn{3}{|l|}{ Doença crônica $(n=1.019)$} \\
\hline Não & 212 & 20,80 \\
\hline Sim & 807 & 79,20 \\
\hline \multicolumn{3}{|l|}{ Estado de saúde $(n=1.026)$} \\
\hline Bom/muito bom & 590 & 57,50 \\
\hline Regular & 358 & 34,90 \\
\hline Ruim/muito ruim & 78 & 7,60 \\
\hline \multicolumn{3}{|l|}{ Possui plano de saúde privado $(\mathrm{n}=1.026)$} \\
\hline Não & 651 & 63,45 \\
\hline Sim & 375 & 36,55 \\
\hline \multicolumn{3}{|l|}{ Serviço de saúde de referência $(n=1.022)$} \\
\hline UBSF* de referência (mais próxima da residência) & 628 & 61,44 \\
\hline Médico/serviço de saúde vinculado a convênio de saúde privado & 140 & 13,70 \\
\hline Consultório médico particular & 94 & 9,20 \\
\hline Hospital/pronto-socorro & 93 & 9,10 \\
\hline Outra UBSF & 48 & 4,70 \\
\hline Outro & 19 & 1,86 \\
\hline
\end{tabular}

UBSF: Unidade Básica de Saúde da Família. 
Quanto à busca por serviços de saúde, quase $2 / 3$ dos entrevistados $(61,4 \%)$ indicaram a UBSF mais próxima de sua residência como o serviço de primeira opção/de referência. Ao estratificar as análises entre UBSF de referência e demais locais de atendimento, os motivos para escolha do serviço se mostraram diferentes (Tabela 2). Enquanto aqueles que preferiam ser atendidos na sua UBSF de referência o faziam principalmente em função da maior proximidade entre residência e o local de atendimento, os demais seguiam seu acompanhamento em outros serviços por estarem habituados, sabendo que lá conseguiriam o atendimento necessário com maior facilidade e resolutividade.

Quanto aos motivos que levaram os usuários a buscar o serviço, o tratamento de doenças (crônicas e agudas) já instauradas foi a principal causa em ambos os grupos. Cabe destacar que apenas pouco mais da metade daqueles que apresentaram quadro de doença crônica buscaram atendimento em seu serviço de referência ( $44 \%$ buscaram atendimento, enquanto 79,2\% relataram apresentar alguma doença crônica).

As Tabelas 3 e 4 descrevem informações referentes à procura de atendimento nos 12 meses anteriores à pesquisa na UBSF mais próxima à moradia dos idosos participantes, isto é, a UBSF considerada como unidade de referência pela gestão municipal em saúde. Dentre os entrevistados, $60,1 \%$ buscaram atendimento no local, com procura maior entre aqueles

Tabela 2. Motivos para escolha e busca do serviço de saúde de referência por idosos da área rural do município de Rio Grande, Rio Grande do Sul, Brasil, 2017 ( $n=1.018$ )

\begin{tabular}{|c|c|c|c|c|c|}
\hline & \multicolumn{4}{|c|}{ Serviço de referência } & \multirow{3}{*}{$\begin{array}{l}\text { Total } \\
\text { n }(\%)\end{array}$} \\
\hline & \multicolumn{2}{|c|}{$\begin{array}{l}\text { UBSF}^{a} \text { de referência } \\
\qquad(n=628)\end{array}$} & \multicolumn{2}{|c|}{$\begin{array}{l}\text { Outro serviço } \\
(\mathrm{n}=390)\end{array}$} & \\
\hline & n (\%) & Posição & n (\%) & Posição & \\
\hline \multicolumn{6}{|l|}{ Motivos para escolha do serviço de referência* } \\
\hline Mais próximo da residência & $519(82,6)$ & $1^{\circ}$ & $25(6,4)$ & $5^{\circ}$ & $544(53,4)$ \\
\hline Serviço que vou habitualmente, pois oferece o tratamento necessário & $167(26,6)$ & $2^{\circ}$ & $205(52,6)$ & $1^{\circ}$ & $372(36,5)$ \\
\hline Gosto/confio no atendimento & $103(16,4)$ & $3^{\circ}$ & $121(31,0)$ & $3^{\circ}$ & $224(22,0)$ \\
\hline Mais fácil/rápido conseguir atendimento & $58(9,2)$ & $4^{\circ}$ & $135(34,6)$ & $2^{\circ}$ & $193(19,0)$ \\
\hline Fica aberto no horário que preciso & $36(5,7)$ & $5^{\circ}$ & $44(11,3)$ & $4^{\circ}$ & $80(7,9)$ \\
\hline Por ser minha UBSF de referência & $17(2,7)$ & $6^{\circ}$ & - & - & $17(1,7)$ \\
\hline Dificuldade em ser atendido pela UBSF de referência & - & - & $07(1,8)$ & $6^{\circ}$ & $7(0,7)$ \\
\hline \multicolumn{6}{|l|}{ Motivos para busca do serviço de referência* } \\
\hline Tratamento de doença crônica & $295(47,0)$ & $1^{\circ}$ & $153(39,2)$ & $1^{\circ}$ & $448(44,0)$ \\
\hline Tratamento de doença aguda & $265(42,2)$ & $2^{\circ}$ & $148(38,0)$ & $2^{\circ}$ & $413(40,6)$ \\
\hline Realização de procedimento & $226(36,0)$ & $3^{\circ}$ & $19(4,9)$ & $5^{\circ}$ & $245(24,1)$ \\
\hline Solicitação/realização de exames & $153(24,4)$ & $4^{\circ}$ & $52(13,3)$ & $4^{\circ}$ & $205(20,1)$ \\
\hline Consulta de revisão/rotina & $112(17,8)$ & $5^{\circ}$ & $81(20,8)$ & $3^{\circ}$ & $193(19,0)$ \\
\hline
\end{tabular}

UBSF: Unidade Básica de Saúde da Família

* Mais de uma resposta por indivíduo.

Tabela 3. Informações relativas à procura por atendimento na UBSF de referência nos 12 meses anteriores à pesquisa por idosos da área rural do município de Rio Grande, Rio Grande do Sul, Brasil, 2017.

\begin{tabular}{|c|c|c|c|c|}
\hline & \multicolumn{2}{|c|}{ Serviço de referência } & \multirow{2}{*}{ Total n (\%) } & \multirow{2}{*}{$\mathbf{p}$} \\
\hline & UBSF de referência $\mathbf{n}(\%)$ & Outro serviço n (\%) & & \\
\hline Buscou atendimento na UBSF de referência nos últimos 12 meses $(n=1.014)$ & $(n=626)$ & $(n=388)$ & & \\
\hline Não & $158(25,2)$ & $247(63,7)$ & $405(39,9)$ & $<0,001$ \\
\hline Sim & $468(74,8)$ & $141(36,3)$ & $609(60,1)$ & \\
\hline Satisfação com o atendimento do local $(n=589)$ & $(n=450)$ & $(n=139)$ & & 0,52 \\
\hline Ruim/muito ruim & $7(1,6)$ & $4(2,9)$ & $11(1,9)$ & \\
\hline Regular & $51(11,3)$ & $10(7,2)$ & $61(10,4)$ & \\
\hline Bom/muito bom & $392(87,1)$ & $125(89,9)$ & $517(87,7)$ & \\
\hline
\end{tabular}

UBSF: Unidade Básica de Saúde da Família. 
Tabela 4. Informações relativas aos motivos de busca e não busca por atendimento na UBSF de referência nos 12 meses anteriores à pesquisa por idosos da área rural do município de Rio Grande, Rio Grande do Sul, Brasil, 2017.

\begin{tabular}{|c|c|c|c|c|c|}
\hline & \multicolumn{4}{|c|}{ Serviço de referência } & \multirow{3}{*}{ Total n (\%) } \\
\hline & \multicolumn{2}{|c|}{ UBSF de referência } & \multicolumn{2}{|c|}{ Outro serviço } & \\
\hline & n (\%) & Posição & n $(\%)$ & Posição & \\
\hline Motivos para busca da UBSF nos últimos 12 meses $(n=609)$ & $(n=468)^{*}$ & & $(n=141)^{*}$ & & \\
\hline Tratamento de doença crônica & $188(40,2)$ & $1^{\circ}$ & $37(26,2)$ & $2^{\circ}$ & $225(37,0)$ \\
\hline Tratamento de doença aguda & $148(31,6)$ & $3^{\circ}$ & $29(20,6)$ & $3^{\circ}$ & $177(29,1)$ \\
\hline Realização de procedimento & $166(35,5)$ & $2^{\circ}$ & $57(40,4)$ & $1^{\circ}$ & $223(36,6)$ \\
\hline Solicitação/realização de exames & $97(20,7)$ & $4^{\circ}$ & $13(9,2)$ & $4^{\circ}$ & $110(18,1)$ \\
\hline Consulta de revisão/rotina & $70(15,0)$ & $5^{\circ}$ & $6(4,3)$ & $5^{\circ}$ & $76(12,5)$ \\
\hline Motivos para a não busca de atendimento na UBSF nos últimos 12 meses $(n=405)$ & $(\mathbf{n}=158)^{*}$ & & $(\mathrm{n}=247)^{*}$ & & \\
\hline Não precisou de atendimento & $133(84,2)$ & $1^{\circ}$ & $87(35,2)$ & $2^{\circ}$ & $220(54,3)$ \\
\hline Buscou atendimento em outro serviço & $13(8,2)$ & $2^{\circ}$ & $104(42,1)$ & $1^{\circ}$ & $117(28,9)$ \\
\hline Encontrou dificuldade em obter atendimento na UBSF na última vez que buscou & $8(5,1)$ & $3^{\circ}$ & $26(10,5)$ & $3^{\circ}$ & $34(8,4)$ \\
\hline
\end{tabular}

UBSF: Unidade Básica de Saúde da Família

* Mais de uma resposta por indivíduo.

Tabela 5. Análise bruta e ajustada da associação de variáveis independentes com a escolha da UBSF como serviço de referência por idosos da área rural do município de Rio Grande, Rio Grande do Sul, Brasil, 2017 ( $n=960$ ).

\begin{tabular}{|c|c|c|c|c|}
\hline \multirow{2}{*}{ Variável } & \multicolumn{2}{|c|}{ Análise bruta } & \multicolumn{2}{|c|}{ Análise ajustada } \\
\hline & RP (IC95\%) & Valor $\mathrm{p}$ & RP (IC95\%) & $p$ \\
\hline \multicolumn{5}{|l|}{ Nível 1 - Fatores Predisponentes } \\
\hline Sexo & & 0,506 & & 0,527 \\
\hline Masculino & $1,03(0,94-1,14)$ & & $1,03(0,94-1,14)$ & \\
\hline Feminino & 1 & & 1 & \\
\hline Idade & & $0,026^{*}$ & & $0,032 *$ \\
\hline 60 a 69 anos & $1,17(1,01-1,35)$ & & $1,16(1,00-1,35)$ & \\
\hline 70 a 79 anos & $1,08(0,92-1,27)$ & & $1,08(0,92-1,27)$ & \\
\hline 80 anos ou mais & 1 & & 1 & \\
\hline Cor da pele & & 0,154 & & 0,194 \\
\hline Branca & 1 & & 1 & \\
\hline Preta/Parda/Amarela/Indígena & $1,12(0,96-1,30)$ & & $1,13(0,96-1,34)$ & \\
\hline \multicolumn{5}{|l|}{ Nível 2 - Fatores Capacitantes } \\
\hline Renda em tercis & & $<0,001^{*}$ & & $<0,001^{*}$ \\
\hline $1^{\circ}$ tercil (menor renda) & $1,43(1,24-1,64)$ & & $1,22(1,05-1,42)$ & \\
\hline $2^{\circ}$ tercil & $1,34(1,16-1,55)$ & & $1,16(1,01-1,34)$ & \\
\hline $3^{\circ}$ tercil (maior renda) & 1 & & 1 & \\
\hline Escolaridade & & $<0,001^{*}$ & & $<0,001^{*}$ \\
\hline 0 a 4 anos & $2,25(1,61-3,61)$ & & $1,94(1,39-2,71)$ & \\
\hline 5 a 8 anos & $1,81(1,27-2,59)$ & & $1,57(1,11-2,22)$ & \\
\hline 9 anos ou mais & 1 & & 1 & \\
\hline Mora sozinho & & 0,703 & & 0,06 \\
\hline Não & 1 & & 1 & \\
\hline Sim & $0,98(0,87-1,10)$ & & $0,89(0,78-1,01)$ & \\
\hline Distância domicílio-UBSF & & $<0,01$ & & 0,02 \\
\hline$<1 \mathrm{~km}$ & $1,27(1,11-1,45)$ & & $1,17(1,03-1,34)$ & \\
\hline 1 a $4,9 \mathrm{~km}$ & $1,12(1,00-1,26)$ & & $1,10(0,98-1,23)$ & \\
\hline $5 \mathrm{~km}$ ou mais & 1 & & 1 & \\
\hline Não soube informar & $1,00(0,86-1,16)$ & & $0,95(0,82-1,10)$ & \\
\hline Possuir plano de saúde & & $<0,001$ & & $<0,001$ \\
\hline Não & 1 & & 1 & \\
\hline $\operatorname{Sim}$ & $0,70(0,62-0,79)$ & & $0,75(0,66-0,84)$ & \\
\hline \multicolumn{5}{|l|}{ Nível 3 - Necessidades em Saúde } \\
\hline Doença Crônica & & 0,952 & & 0,55 \\
\hline Não & 1 & & 1 & \\
\hline Sim & $1,00(0,88-1,12)$ & & $0,96(0,86-1,09)$ & \\
\hline Estado de Saúde & & $0,27^{*}$ & & $0,99 *$ \\
\hline Bom/Muito bom & 1 & & 1 & \\
\hline Regular & $1,06(0,96-1,18)$ & & $1,00(0,90-1,11)$ & \\
\hline Ruim/Muito ruim & $1,06(0,88-1,27)$ & & $0,99(0,83-1,20)$ & \\
\hline
\end{tabular}

RP: razão de prevalência; IC95\%: intervalo de confiança de 95 \%; UBSF: Unidade Básica de Saúde da Família.

* Valor p de tendência linear. 
que consideravam o local como seu serviço de referência ( $75 \%$ versus $36 \%$ ). Dentre aqueles que procuraram o serviço, $87,7 \%$ avaliaram o atendimento positivamente, não havendo diferença significativa entre os grupos.

Os motivos que levaram os usuários a buscar atendimento na respectiva UBSF foram diferentes entre aqueles que consideravam a unidade como sua primeira opção de serviço e aqueles que tinham outro local como referência. Enquanto os primeiros procuravam mais a unidade de forma geral (tratamento de doenças, revisão, exames), os demais buscavam a UBSF mais frequentemente para realização de procedimentos.

Dentre aqueles que não buscaram a UBSF nos últimos 12 meses $(\mathrm{n}=405)$, os motivos para isso se mostraram diferentes entre os dois grupos. Enquanto os que consideravam a UBSF como primeira opção de serviço relataram não perceber necessidade em buscar algum tipo de atendimento $(84,2 \%$ versus $35,2 \%)$, os demais relataram como motivo a preferência por outro local, que consideravam mais resolutivo para obter a consulta necessária (42,1\% versus 8,2\%).

A Tabela 5 apresenta os fatores associados à escolha da UBSF como serviço de referência, demonstrando que, quanto menores a idade, renda, escolaridade e distância da residência em relação à unidade, maior a probabilidade do idoso considerar a UBSF mais próxima como serviço de referência. Além disso, possuir plano de saúde privado diminuiu a probabilidade de escolha em $25 \%$, enquanto características relacionadas às necessidades em saúde dos participantes não se mostraram estatisticamente associadas à escolha (Tabela 5).

\section{DISCUSSÃO}

O presente estudo identificou que quase $2 / 3(61,4 \%)$ dos idosos moradores da área rural do município de Rio Grande consideraram a UBSF mais próxima da residência como serviço de saúde de referência. Quanto menores a idade, escolaridade, renda e distância da UBSF, maior a probabilidade do serviço de APS ser escolhido como primeira opção pelos usuários. O percentual de idosos que considerou a APS como serviço usual se mostrou elevado quando comparado a outro estudo realizado no Brasil, inclusive no que diz respeito especificamente ao grupo de idosos e população rural (36,2\% e 49,2\%, respectivamente) $)^{22}$.

A definição de um serviço de referência tem se mostrado positivamente associada a melhorias na condição de saúde do usuário e maior eficiência do sistema, uma vez que o acompanhamento longitudinal do indivíduo previne o aparecimento e evolução de doenças, evitando a sobrecarga dos serviços de emergência e de consultas com especialistas por causas evitáveis ${ }^{15,22,23}$. Além disso, tal definição possibilita o desenvolvimento de uma relação de confiança entre equipe de saúde e usuário, fortalecendo a adesão ao tratamento ${ }^{2,3,24}$.

Seguindo a categorização desenvolvida por Aday e Andersen ${ }^{21}$, as principais necessidades em saúde percebidas pelos idosos como motivos para busca tanto das UBSF quanto dos demais serviços de referência foram quadros de doenças já instauradas (doenças crônicas e agudas), mostrando um perfil semelhante de necessidades entre ambos os grupos. A maior procura dos serviços públicos de APS pode ser reflexo de esforços governamentais para anualmente imunizar idosos contra influenza, em campanhas que ultrapassaram a meta de $97 \%$ dos idosos vacinados em 2018, chegando a 99,39\% de cobertura em $2019^{25}$.

Além disso, na literatura, ser portador de doença crônica tem se destacado como fator associado a maior procura por atendimento em saúde ${ }^{5,9,23}$. Todavia, como já apontado em outro estudo conduzido no Brasil ${ }^{14}$, o percentual de utilização dos serviços ainda não está adequado às necessidades dos usuários, uma vez que, dentre os participantes que relataram ter algum quadro crônico, $40 \%$ não buscaram atendimento no último ano. 
Considerando os fatores capacitantes, merece destaque a semelhança entre os grupos, embora o perfil de atendimento dos serviços seja diferente. Enquanto serviços de pronto atendimento e consultórios médicos particulares/conveniados têm caráter mais responsivo, geralmente atendendo apenas a demandas de usuários já enfermos, os serviços de APS têm por objetivo desenvolver, além do tratamento e da reabilitação, práticas de prevenção à doença, promoção da saúde e participação popular².

Essas últimas finalidades, porém, não se sobressaíram entre os motivos para procurar as UBSF, o que pode ser reflexo de crenças e comportamentos em saúde da população estudada, por exemplo por desconhecimento ou desengajamento de usuários e equipe profissional quanto às finalidades mais amplas da APS, não percebidas como prioridade ${ }^{12,23,26}$. $\mathrm{O}$ alto percentual de idosos que relataram não ter buscado atendimento para doenças crônicas e a forma como lidaram com a enfermidade e seus possíveis estigmas também podem ser considerados parte destas crenças e comportamentos ${ }^{15,21,24}$.

A oferta gratuita de serviços pelo sistema público de saúde brasileiro permite que todos tenham acesso à atenção mais facilmente. Todavia, ter plano de saúde privado ou melhores condições econômicas mostrou-se associado à escolha de outros serviços de saúde como primeira opção, o que pode significar, como já identificado na literatura, que, tendo meios de acessar serviços mais custosos, os idosos tendem a buscar outros locais de atendimento ${ }^{17,18,26}$.

Quanto à escolaridade, a associação encontrada pode estar relacionada a um maior autoconhecimento geral e em saúde, bem como a taxas mais elevadas de automedicação (permitindo melhor identificação do serviço adequado para o quadro de saúde ou diminuindo a percepção de necessidade de acompanhamento) ${ }^{14,26,27}$.

Outro fator capacitante refere-se à estrutura e organização dos serviços de saúde. Enquanto a distância foi um aspecto decisivo para a escolha das UBSF como serviço de referência, outros elementos - como resolutividade ("oferece o tratamento que necessito"), facilidade e rapidez na obtenção do atendimento e confiança no tratamento/profissional - estiveram presentes nas respostas daqueles que buscam outros locais. A influência da distância até o local de atendimento desejado já foi alvo de várias pesquisas, mostrando que maiores distâncias levam a uma menor utilização dos serviços ${ }^{3,13,17}$, principalmente quando se trata de indivíduos com baixa mobilidade, com poucas opções de serviços disponíveis e pouco ou nenhum acesso a meios de transporte ${ }^{4,15,28}$. Contudo, a proximidade não é o único fator de garantia de acesso, uma vez que moradores de áreas rurais podem estar dispostos a percorrer maiores distâncias quando julgam que não receberiam o atendimento mais adequado às suas necessidades perto de casa, mesmo que o deslocamento signifique maiores custos e maior desgaste físico e emocional ${ }^{10,17,28}$.

Quanto a fatores predisponentes, encontrou-se associação apenas com a idade. Idosos mais jovens podem apresentar melhor mobilidade e capacidade funcional, o que permite a busca ativa por atendimento para além das visitas domiciliares; já indivíduos mais velhos podem requerer atendimentos mais específicos e especializados, indisponíveis no nível primário de atenção ${ }^{5,14,29}$.

Motivos que levam a não buscar um serviço também são aspectos importantes, que complementam a percepção de organização do local por parte do usuário. Entre aqueles que veem a UBSF como primeira opção de atendimento, a principal causa para não a buscar foi considerar que não havia necessidade, realidade semelhante à identificada por outros estudos 5 . Porém, a percepção de não necessidade pode ser questionada, visto se tratar de um grupo populacional que precisa de maiores cuidados e, consequentemente, requer revisões qualificadas mais frequentes para avaliar seu real estado de saúde. Os princípios da ESF se mostram bastante pertinentes neste contexto, uma vez que esta prima pela busca ativa e acompanhamento longitudinal através de acompanhamento familiar e visitas domiciliares feitas por agentes comunitários de saúde e equipe técnica ${ }^{2,18}$. 
Deve-se considerar que, como pesquisa transversal, o presente estudo não intenciona investigar relações de causa e efeito entre as variáveis apresentadas, visto que o delineamento utilizado está suscetível à causalidade reversa. Além disso, cabe ressaltar como limitações: (1) características referentes à organização do serviço foram coletadas apenas através dos usuários, não tendo ocorrido contato direto com os serviços de atendimento; (2) as medidas referentes à distância entre residência e UBSF também foram coletadas através do relato dos entrevistados, gerando dados não tão precisos quanto os georreferenciados e perda elevada de respostas, visto que alguns idosos não tinham essa informação.

Considerando que o acesso está fortemente relacionado às percepções individuais ${ }^{15}$ (isto é, quão fácil o usuário percebe ser o acesso), a complementação dos dados por diferentes fontes enriqueceria os resultados. Ainda assim, o foco exclusivo no usuário é de grande relevância e não inviabiliza os achados expostos, uma vez que as necessidades em saúde percebidas pelo usuário permitem antecipar demandas e são uma das bases para elaborar políticas públicas ${ }^{29}$.

Por fim, conforme evidenciado, a ESF tem conseguido alcançar grande parte da população rural idosa, principalmente grupos em situação de vulnerabilidade, indo ao encontro de um sistema de saúde público equitativo. Todavia, apesar de ter acesso às UBSF, 133 do grupo estudado busca outros serviços para acompanhamento. Ou seja, mesmo com uma cobertura de $100 \%$ pela ESF, estrutura social, fatores capacitantes (como condição econômica) e crenças sobre saúde ainda definem o padrão de escolha do serviço a ser procurado.

Desta forma, destaca-se a necessidade de continuar a fortalecer a rede de cuidado daqueles que apresentam maiores necessidades em saúde, direcionando a atenção aos perfis de usuários que não buscaram o serviço. Pode-se desenvolver atividades de caráter preventivo e de promoção à saúde direcionadas àqueles que não percebem necessidade de acompanhamento, bem como adequar a estrutura e a organização para trazer à UBSF os que buscam outro local de atendimento. Para isso, novas avaliações por parte de gestores, pesquisadores e profissionais são pertinentes, a fim de ampliar os conhecimentos sobre o tema em diferentes contextos e averiguar a adequação e a qualidade do atendimento oferecido.

\section{REFERÊNCIAS}

1. International Conference on Primary Health Care; 1978 Sept 6-12; Alma- Ata, URSS. Declaration of Alma-Ata. Geneva: WHO; 1978 [citado 17 dez 2019]. Disponível em: https://www.who.int/publications/almaata_declaration_en.pdf

2. Ministério da Saúde (BR). Portaria n 2.436, de 21 de setembro de 2017. Aprova a Política Nacional de Atenção Básica, estabelecendo a revisão de diretrizes para a organização da Atenção Básica, no âmbito do Sistema Único de Saúde (SUS). Diário Oficial da União. 22 ago 2017; Seção 1:68.

3. Fontenelle LF, Camargo MBJ, Bertoldi AD, Gonçalves H, Maciel ELN, Barros AJD. Utilização das unidades básicas de saúde da ESF conforme a cobertura por plano de saúde. Rev Saude Publica. 2018;52:55. https://doi.org/10.11606/S1518-8787.2018052000383

4. Garbaccio JL, Tonaco LAB, Estêvão WG, Barcelos BJ. Envelhecimento e qualidade de vida de idosos residentes da zona rural. Rev Bras Enferm. 2018;71Supl 2:776-84. https://doi.org/10.1590/0034-7167-2017-0149

5. Silva KF, Pucci VR, Weiller TH, Mayer BLD, Concatto MEP. O acesso do idoso na Atenção Primária à Saúde: uma revisão integrativa. Rev APS. 2018;21(1):122-33. https://doi.org/10.34019/1809-8363.2018.v21.15911

6. Ministério da Saúde (BR). Histórico de Cobertura da Estratégia Saúde da Família. Brasília, DF; 2017 [citado 17 dez 2019]. Disponível em: https://egestorab.saude.gov.br/paginas/acessoPublico/relatorios/relHistoricoCoberturaAB.xhtml

7. Paim J, Travassos C, Almeida C, Bahia L, Macinko J. O sistema de saúde brasileiro: história, avanços e desafios. Lancet. 6736(11):11-31 (Série Saúde no Brasil). 2011;1:11-31. https://doi.org/10.1016/S0140-6736(11)60054-8 
8. Arruda NM, Maia AG, Alves LC. Desigualdade no acesso à saúde entre as áreas urbanas e rurais do Brasil: uma decomposição de fatores entre 1998 a 2008. Cad Saude Publica. 2018;34(6):e00213816. https://doi.org/10.1590/0102-311X00213816

9. Almeida APSC, Nunes BP, Duro SMS, Facchini LA. Determinantes socioeconômicos do acesso a serviços de saúde em idosos: revisão sistemática. Rev Saude Publica. 2017;51:50. https://doi.org/10.1590/S1518-8787.2017051006661

10. Szwarcwald CL, Damacena GN, Souza Júnior PRB, Almeida WS, Malta DC. Percepção da população brasileira sobre a assistência prestada pelo médico. Brasil, 2013. Cienc Saude Colettiva. 2016;21(2):339-49. https://doi.org/10.1590/1413-81232015212.19332015

11. Travassos C, Martins M. Uma revisão sobre os conceitos de acesso e utilização de serviços de saúde. Cad Saude Publica. 2004;20 Supl 2:S190-8. https://doi.org/10.1590/S0102-311X2004000800014

12. Shimizu HE, Trindade JS, Mesquita MS, Ramos MC. Avaliação do Índice de Responsividade da Estratégia Saúde da Família da zona rural. Rev Esc Enferm USP. 2018;52:e03316. https://doi.org/10.1590/s1980-220x2017020203316

13. Garnelo L, Lima JG, Rocha ESC, Herkrath FJ. Acesso e cobertura da Atenção Primária à Saúde para populações rurais e urbanas na região norte do Brasil. Saude Debate. 2018;42 № Espec 1:81-99. https://doi.org/10.1590/0103-11042018s106

14. Travassos C, Viacava F. Acesso e uso de serviços de saúde em idosos residentes em áreas rurais, Brasil, 1998 e 2003. Cad Saude Publica. 2007;23(10):2490-502. https://doi.org/10.1590/S0102-311X2007001000023

15. Pedraza DF, Nobre AMD, Albuquerque FJB, Menezes TN. Acessibilidade às Unidades Básicas de Saúde da Família na perspectiva de idosos. Cienc Saude Coletiva. 2018;23(3):923-33. https://doi.org/10.1590/1413-81232018233.11702016

16. Bortolotto CC, Loret de Mola C, Tovo-Rodrigues L. Qualidade de vida em adultos de zona rural no Sul do Brasil: estudo de base populacional. Rev Saude Publica. 2018;52 Supl 1:4s. https://doi.org/10.11606/s1518-8787.2018052000261

17. Gustafsdottir SS, Fenger K, Halldorsdottir S, Bjarnason T. Social justice, access and quality of healthcare in an age of austerity: users' perspective from rural Iceland. Int J Circumpolar Health. 2017;76(1):1347476. https://doi.org/10.1080/22423982.2017.1347476

18. Macinko J, Andrade FB, Souza-Júnior PRB, Lima-Costa MF. Primary care and healthcare utilization among older Brazilians (ELSI-Brazil). Rev Saude Publica. 2018;52 Supl 2:6s. https://doi.org/10.11606/s1518-8787.2018052000595

19. Instituto Brasileiro de Geografia e Estatística. Censo Demográfico, 2010. Rio de Janeiro: IBGE; 2011.

20. Victora CG, Hutlly SR, Fuchs SC, Olinto MT. The role of conceptual frameworks in epidemiological analysis: a hierarchical approach. Int J Epidemiol. 1997;26(1):224-7. https://doi.org/10.1093/ije/26.1.224

21. Aday LA, Andersen R. A framework for the study of access to medical care. Health Serv Res. 1974 [citado 18 dez 2019];9(3):208-20. Disponível em: https://www.ncbi.nlm.nih.gov/pubmed/4436074

22. Dourado I, Medina MG, Aquino R. The effect of the Family Health Strategy on usual source of care in Brazil: data from the 2013 National Health Survey (PNS 2013). Int J Equity Health. 2016;15(1):151. https://doi.org/10.1186/s12939-016-0440-7

23. Veras RP, Oliveira M. Envelhecer no Brasil: a construção de um modelo de cuidado. Cienc Saude Coletiva. 2018;23(6):1929-36. https://doi.org/10.1590/1413-81232018236.04722018

24. Gontijo TL, Duarte AGS, Guimarães EAA, Silva J. Avaliação da atenção primária: o ponto de vista de usuários. Saude Debate. 2017;41(114):741-52. https://doi.org/10.1590/0103-1104201711406

25. Ministério da Saúde (BR), DATASUS. Sistema de Informações do Programa Nacional de Imunizações. Brasília, DF; 2018 [citado 18 dez 2019]. Disponível em: http://sipni.datasus.gov.br/si-pni-web/faces/relatorio/consolidado/vacinometrolnfluenza.jsf

26. Araújo LUA, Gama ZAS, Nascimento FLA, Oliveira HFV, Azevedo WM, Almeida Júnior HJB. Avaliação da qualidade da atenção primária à saúde sob a perspectiva do idoso. Cienc Saude Coletiva. 2014;19(8):3521-32. https://doi.org/10.1590/1413-81232014198.21862013

27. Arrais PSD, Fernandes MEP, Dal Pizzol TS, Ramos RL, Mengue SS, Tavares NUL, et al. Prevalência da automedicação no Brasil e fatores associados. Rev Saude Publica. 2016;50 Supl 2:13s. https://doi.org/10.1590/s1518-8787.2016050006117 
28. Stentzel U, Piegsa J, Fredrich D, Hoffmann W, Berg N. Accessibility of general practitioners and selected specialist physicians by car and by public transport in a rural region of Germany. BMC Health Serv Res. 2016;16(1):587. https://doi.org/10.1186\%2Fs12913-016-1839-y

29. Antunes JLF, Chiavegatto Filho ADP, Duarte YAO, Lebrão ML. Desigualdades sociais na autoavaliação de saúde dos idosos da cidade de São Paulo. Rev Bras Epidemiol. 2018;21 Supl 2:e180010. https://doi.org/10.1590/1980-549720180010.supl.2

Contribuição dos Autores: Concepção e planejamento do estudo: LSF, SSP, RDM.Coleta, análise e interpretação dos dados: LSF. Elaboração ou revisão do manuscrito: LSF, LRM, SSP, RDM. Aprovação da versão final: LSF, LRM, SSP, RDM. Responsabilidade pública pelo conteúdo do artigo: LSF, LRM, SSP, RDM.

Conflito de Interesses: Os autores declaram não haver conflito de interesses. 